\title{
TAMAÑO, CONCENTRACIÓN Y RIESGO DE LA BANCA EN MÉXICO (1997-2002)
}

\author{
Jesús Bravo Pliego*
}

Tecnológico de Monterrey, Campus Ciudad de México

José Antonio Núñez Mora

Tecnológico de Monterrey, Campus Ciudad de México

Alejandro Segundo Valdés

Universidad Autónoma Metropolitana-Azcapotzalco

(Recibido 20 de febrero 2003, aceptado 28 de mayo 2003)

\section{Resumen}

Este artículo analiza empíricamente la relación entre riesgo y tamaño para el caso del sistema bancario Mexicano. El uso de observaciones de corte transversal permite obtener indicadores de tamaño, riesgo y concentración de cada banco. Con la agregación de estas observaciones a través de series de tiempo, también construímos indicadores de tamaño, riesgo y concentración. Finalmente, empleando datos panel podemos combinar en nuestro estudio econométrico aspectos de las series de tiempo con algunas características particulares de cada banco.

\section{Abstract}

This paper analyzes empirically the relationship between risk and size in the Mexican Banking System. Cross section data permit us to get size, risk and concentration indicators for each bank. With time series data aggregation, we make up the size, risk and concentration indicators. Finally, with panel data we combine aspects of time series and particular characteristics of each bank in our econometric study.

Clasificación JEL: C21, C23, G21

Palabras clave: Riesgo, Concentración, Series de Tiempo, Corte transversal, Datos panel

* Departamento de Economía, Tecnológico de Monterrey, Campus Ciudad de México. Calle del Puente 222, oficinas 3, segundo piso, Col. Ejidos de Huipulco, Deleg. Tlalpan, C.P. 14380, México, D.F. Teléfono +52 (55) 54832240. Correo electrónico: jesus.bravo@itesm.mx

Los autores desean agradecer a dos dictaminadores anónimos sus valiosas observaciones. 


\section{Introducción}

Es conocida la afirmación de que un mayor nivel de concentración bancaria incrementa significativamente el riesgo agregado del sistema bancario. Muchas veces se asume que existe correlación entre el tamaño del banco y el riesgo al que está expuesto en sus operaciones. En los últimos años la literatura sobre teoría bancaria ha propuesto que la relación entre estas dos variables no es del todo clara. En efecto, mientras una corriente señala que el tamaño de los bancos está relacionado positivamente con el riesgo sistemático que asumen los mismos, otra corriente indica que las dos variables están correlacionadas negativamente. La segunda hipótesis se basa en la teoría del portafolio, en tanto que la primera toma como punto de partida la hipótesis conocida como too big to fail.

El objetivo de este documento consiste en probar cuál de estas hipótesis es válida para el sector bancario de México. Es decir, aquí se estudia empíricamente la relación entre el tamaño de los bancos y el riesgo involucrado en sus operaciones de mercado. El estudio de la relación entre estas dos variables ya ha sido hecho para otros países, los trabajos de Demsetz y Strahan (1995), y Chumacera y Langoni (2001) son buenos ejemplos de ello.

El sector bancario en México ha sufrido cambios estructurales en los últimos años, a lo largo de dos décadas los bancos comerciales fueron estatizados, después reprivatizados $\mathrm{y}$, posteriormente, se vieron envueltos en una crisis de deuda. Finalmente, los bancos fueron sometidos a la apertura externa e internacionalización, de tal forma que la mayoría de los bancos privados en el país tienen, en la actualidad, altos porcentajes de inversión extranjera.

Cada uno de estos procesos ha sido complejo y ha influido de manera importante en la estructura del sector bancario nacional. Entre las principales variables que han sido continuamente modificadas están la estructura de capital y la composición de la deuda del sector. En este sentido, estudiar la relación entre el tamaño y el riesgo que asume cada banco resulta ser una tarea complicada y, en ocasiones, engañosa ya que las series de datos disponible presentan muchas deficiencias.

El trabajo está organizado de la siguiente forma. En la sección dos se examina la relación teórica entre el tamaño del banco y el riesgo existente en el sector. En la sección tres se realiza el estudio empírico para el caso mexicano. Finalmente, en la sección cuatro se presentan las conclusiones.

\section{Relación entre tamaño y riesgo}

\subsection{Relación negativa}

En la teoría del portafolio es bien conocido el argumento que indica que la diversificación de las inversiones reduce el riesgo. Además, se sabe que el proceso de diversificación se hace más intenso cuando el tamaño del portafolio es más grande. Presumiblemente, un banco tendrá portafolios mayores en la medida en que el banco sea más grande. Por esta razón, la relación entre el tamaño y el riesgo, que se establece a través de esta teoría, es negativa.

Por otra parte, desde el punto de vista estadístico, la relación negativa entre estas dos variables puede sostenerse por medio del Teorema del Límite Central. En efecto, de acuerdo a Chumacera y Langoni (2001), si se define a 
$Y$ como el total de activos y a $D$ como el total de deudas, entonces se puede proponer el siguiente modelo:

$$
Y=\sum_{j=1}^{n} Y_{j}
$$

donde $Y_{j}=\mu+\epsilon_{j}, \epsilon_{j}$ es una variable aleatoria $i . i . d$. con $\left(0, \sigma_{\epsilon}^{2}\right)$ y $D=n d$. Aquí $d$ expresa el rendimiento bruto que se paga por un depósito de $\$ 1 ; Y_{j}$ es el retorno bruto (incierto) que se obtiene al prestar $\$ 1$. En este modelo se supone que hay $n$ proyectos. Se considera que un banco incurrirá en bancarrota cuando el total de sus deudas $(D)$ sobrepasa el valor de sus activos $(Y)$. Entonces, tiene sentido definir la probabilidad de bancarrota $\operatorname{Pr}(Y<D)$. A partir de $(1)$ se obtiene (Chumacera y Langoni. 2001):

$$
\lim _{n \rightarrow \infty} \operatorname{Pr}(Y<D)=0 .
$$

La forma de (1) permite definir otro modelo. Si se considera que existe un riesgo agregado, el cual es igual para todos los proyectos, entonces el modelo se puede escribir como:

$$
Y=\sum_{j=1}^{n} Y_{j}
$$

donde $Y_{j}=\mu+\epsilon_{j}+v, v$ es una variable aleatoria $i . i . d$. con $\left(0, \sigma_{v}^{2}\right)$ y $D=n d$. Por el desarrollo de (3) se puede obtener el siguiente resultado:

$$
0<\lim _{n \rightarrow \infty} \operatorname{Pr}(Y<D)=\Phi\left(\frac{d-\mu}{\sigma_{v}}\right)<\frac{1}{2}
$$

En esta expresión $\Phi$ representa la función de distribución acumulada de la normal estándar. Nótese que si la volatilidad del riesgo agregado es suficientemente grande, la relación inversa entre riesgo y tamaño no desaparece, pero se debilita.

\subsection{Relación positiva}

En los últimos años la literatura sobre teoría bancaria se ha desarrollado sobre la base de modelos con fundamentos microeconómicos. Una de esas ideas es el argumento llamado too big to fail. Originalmente, el argumento se desarrolló en la literatura de seguros y tiene que ver con la asimetría del trato de las autoridades que regulan la relación entre los bancos y otros participantes en el mercado. El argumento básico es el siguiente: Debido a la existencia de seguros de depósitos surge un riesgo moral, en consecuencia, los bancos más grandes tienen el incentivo de tomar posiciones de riesgo mayores que los bancos más pequeños; ya que la bancarrota de los bancos mayores es más temida por las autoridades regulatorias. De esta forma, las deudas de dichos bancos está garantizada por las propias autoridades. De hecho, los bancos grandes perciben esta situación y por ello toman posiciones de riesgo más altas.

En la práctica, el argumento teórico too big to fail ha sido sustituido en algunos países y se ha convertido en una política de administración por parte de 
los sistemas financieros. En los Estados Unidos, la política too big to fail se puso en práctica en la década de los setenta, intensificándose en los siguientes diez años (Boyd y Gertler, 1994). Este tipo de política significa una administración suave, en el mejor de los casos, por parte de los reguladores bancarios y les permite a los bancos de mayor tamaño adquirir deudas y diversificar inversiones sin que sean necesariamente más eficientes en su operación. La política en cuestión tiende por definición a fomentar las operaciones de mayor riesgo por parte de los bancos más grandes.

En el caso de México, los acontecimientos de los últimos años imponen la necesidad de explorar esta hipótesis. En efecto, las trasformaciones ocurridas en el sistema financiero y bancario en el país en los años pasados, indican que los procesos de desregulación y apertura financiera son un hecho. Más aún, la quiebra virtual de los bancos privados y su rescate financiero con cargo a las finanzas públicas en la mitad de la década de los noventa, permiten sospechar que la nueva banca en México puede operar con márgenes de riesgo mayores.

\section{Datos y resultados empíricos}

El problema consiste en evaluar empíricamente la relación que existe entre tamaño, concentración y riesgo en el sistema bancario mexicano. Para ello se deben definir las variables adecuadas. Se elaboró una base de datos con información obtenida de la Comisión Nacional Bancaria y de Valores (CNBV) y del Banco de México (BM). La serie es trimestral y cubre el período entre los años de 1997 y 2002.

Se definen las siguientes variables:

- Como es común en la literatura, para medir el tamaño de un banco se toma el logaritmo natural de su cartera total $(Y)$. Es decir, el tamaño de un banco $i$ está definido por $y_{i}=\ln Y_{i}$.

- $D_{i}$ es la cartera vencida del banco $i$.

- Medidas de Concentración. Se usan dos medidas: la primera de ellas es el índice de Herfindahl, $H$; la otra es la participación del banco $i$ en el total de los préstamos del sistema bancario, $S_{i}$.

- Medidas de Riesgo. Se definen dos medidas para el riesgo, una de ellas es expost y la otra es ex-ante. La medida ex-post es la razón entre la cartera vencida y la cartera total de cada banco $i, P_{i}$. La segunda medida consiste en una medida ponderada de la "calificación" de la cartera de cada banco. Con la calificación de la cartera existe un problema con los datos originales, una parte de la base de datos tiene la etiqueta "exceptuada", otra (muy pequeña en proporción) aparece con la etiqueta "no calificada", lo que resta de la información sí tiene calificación.

De la parte de la cartera que tiene calificación hacemos la siguiente clasificación para medir el riesgo: A la cartera con riesgo mínimo se le asigna el número 0.2 ; las carteras con riesgo bajo y medio tienen los números 0.4 y 0.6 respectivamente. Finalmente, a la cartera irrecuperable se le asignó el número 1. Lo que hacemos con esta escala es elaborar una medida de riesgo ponderada de los niveles de riesgo en nuestra escala (de 0.2 a 1 ) y las proporciones de cartera en cada nivel de riesgo. Ésta es la medida de riesgo ex-post, $R_{i}$. 
- Las series de datos macroeconómicos se obtuvieron a partir de la base de datos de Banco de México.

\subsection{Análisis empírico}

El análisis empírico tiene tres partes: a) Corte Transversal. Aquí se usa como ejemplo el segundo trimestre del año 2002, de hecho los resultados son muy parecidos trimestre a trimestre; b) Series de Tiempo. Usamos los datos del último trimestre del año de 1997 hasta el segundo trimestre de 2002 y c) Datos Panel. Se usan la misma serie de datos del inciso anterior pero para cada uno de los bancos.

\subsubsection{Corte transversal}

Se estimaron ecuaciones del tipo $P=\beta_{0}+\beta_{1} y+\beta_{2} y^{2}+\beta_{3} S+u$ y $R=\beta_{0}+\beta_{1} y+$ $\beta_{2} y^{2}+\beta_{3} S+u$, para cada una de nuestras variables de riesgo. Aquí las variables dependientes son el tamaño del banco $(y)$ y la medida de concentración $S$. No se encontró ninguna relación significativa entre el riesgo y las otras variables existentes. Las gráficas 1-4 reflejan esta situación, ya que podemos observar que no existe correlación entre las medidas de riesgo y el tamaño de los bancos $(y)$, ni con la participación de cada uno de ellos en el total de los préstamos totales $(S)$.

\begin{tabular}{|c|c|c|c|c|}
\hline \multicolumn{5}{|c|}{$P=\beta_{0}+\beta_{1} y+\beta_{2} y^{2}+\beta_{3} S+u$} \\
\hline Variable & Coeficiente & Error Estándar & Estadístico $t$ & P-Value \\
\hline Constante & 0.084354 & 0.055607 & 1.516966 & 0.1418 \\
\hline$y$ & -0.005265 & 0.008663 & -0.607771 & 0.5488 \\
\hline$y^{2}$ & -0.003952 & 0.003364 & -1.174799 & 0.2511 \\
\hline$S$ & 0.742977 & 0.630973 & 1.177511 & 0.2501 \\
\hline \multicolumn{5}{|c}{$R^{2}=0.051939$} \\
\hline
\end{tabular}

\begin{tabular}{|c|c|c|c|c|}
\hline \multicolumn{5}{|c|}{$R=\beta_{0}+\beta_{1} y+\beta_{2} y^{2}+\beta_{3} S+u$} \\
\hline Variable & Coeficiente & Error Estándar & Estadistico $t$ & P-Value \\
\hline Constante & 0.286965 & 0.037176 & 7.719146 & 0 \\
\hline$y$ & -0.005537 & 0.007601 & -0.728495 & 0.4731 \\
\hline$y^{2}$ & -0.000748 & 0.002393 & -0.312470 & 0.7573 \\
\hline$S$ & 0.348881 & 0.410685 & 0.849511 & 0.4037 \\
\hline \multicolumn{5}{|c}{$R^{2}=0.022079$} \\
\hline
\end{tabular}

Notas: La muestra es del segundo trimestre del 2002, el número de observaciones es de 29 y las desviaciones estándar se calcularon usando la matriz de varianzas y covarianzas de White. 


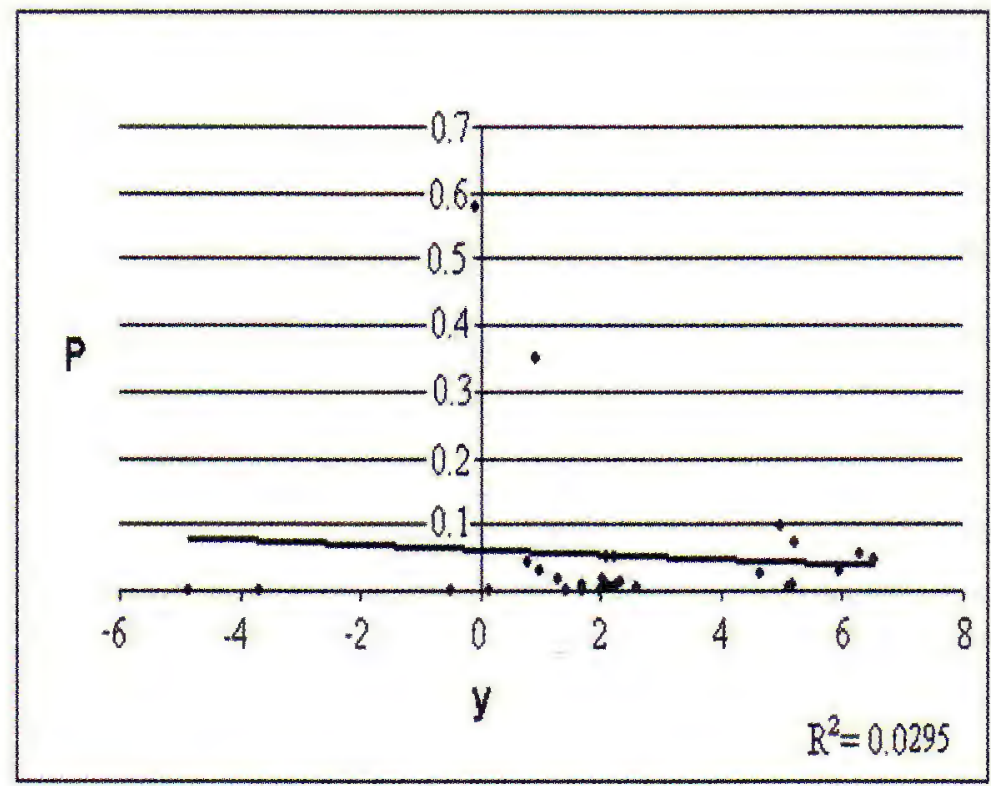

Gráfico 1. Cártera vencida vs. Tamaño.

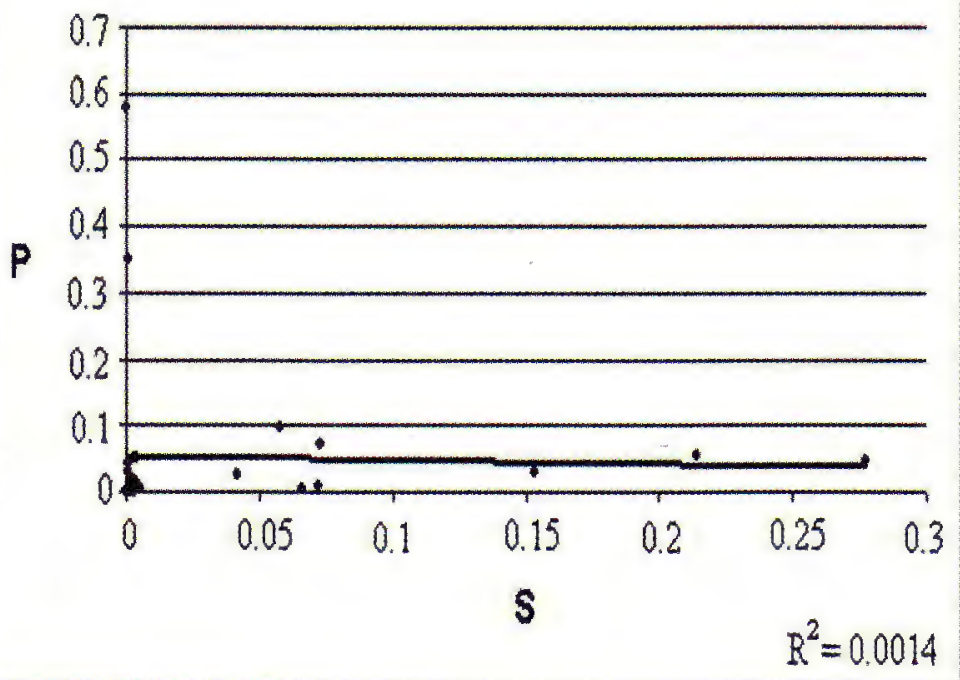

Gráfico 2. Cártera vencida vs. Participación. 


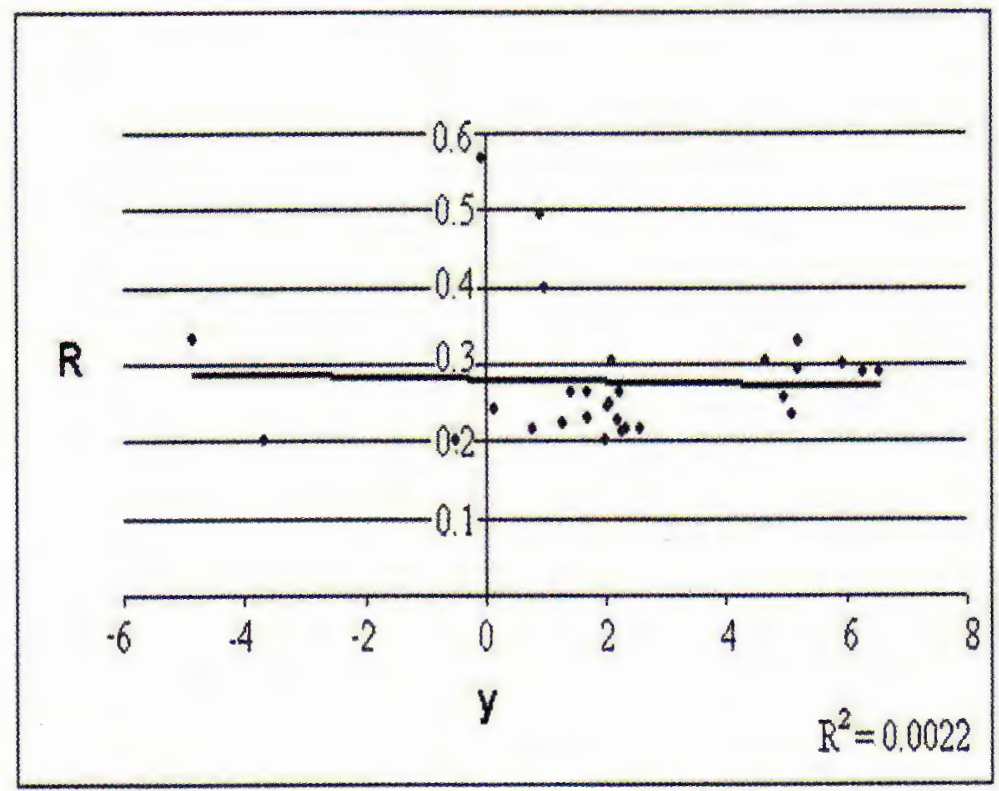

Gráfico 3. Índice de calificación vs. Tamaño.

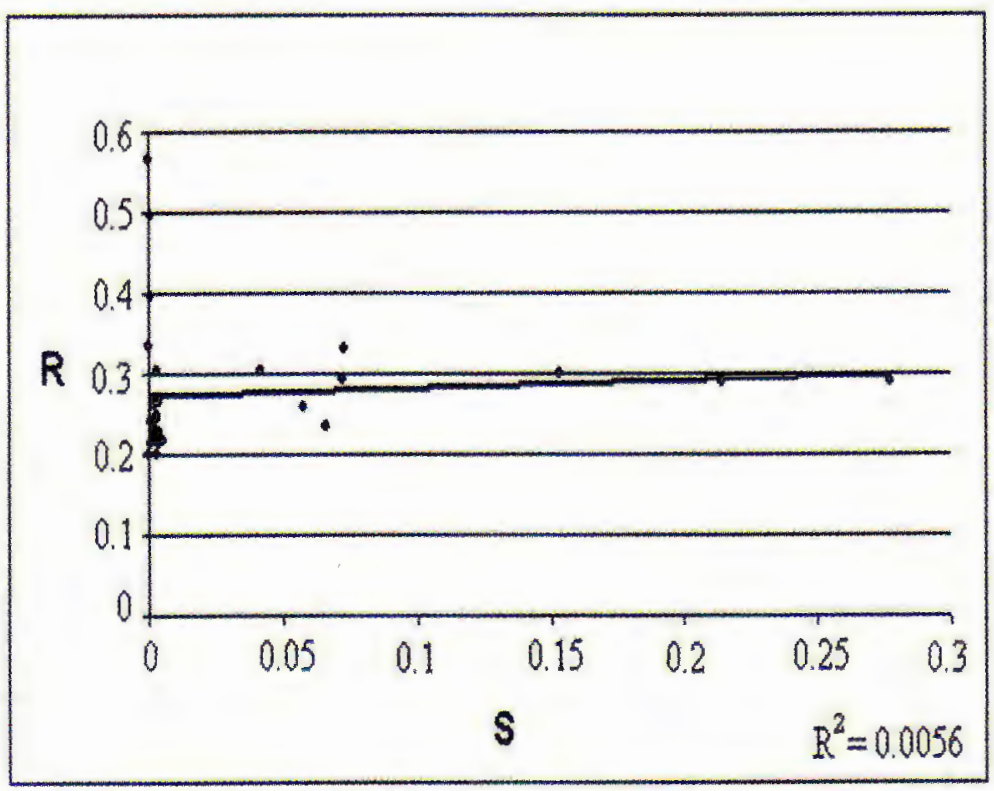

Gráfico 4. Índice de calificación vs. Participación. 


\subsubsection{Series de Tiempo}

En este caso, las gráficas 5 y 7 sugieren que las medidas de riesgo y el tamaño de los bancos tienen una correlación positiva, mientras que las gráficas 6 y 8 indican que las medidas de riesgo tienen una correlación negativa con el índice de concentración de Herfindahl. Sin embargo, no existe un patrón robusto de comportamiento entre las variables de riesgo, tamaño y concentración. ${ }^{1}$ Por lo anterior, es necesario hacer un análisis más detallado incluyendo en las estimaciones un conjunto de variables exógenas (macrocconómicas), tales como: tasa de crecimiento del PIB $(R g)$, inflación $(\pi)$ y tasa de interés de los CETES a 28 días $(I)$. Se estimaron los modelos con las dos medidas de riesgo y se encontró que ninguna variable es estadísticamente significativa.

\begin{tabular}{|c|c|c|c|c|}
\hline \multicolumn{5}{|c|}{$P_{t}=\beta_{0}+\beta_{1} y_{t}+\beta_{2} y_{t}^{2}+\beta_{3} H_{t}+\beta_{4} R g_{t}+\beta_{5} \pi_{t}+\beta_{6} I_{t}+\beta_{7} P_{t-1}+u_{t}$} \\
\hline Variable & Coeficiente & Error Estándar & Estadístico $t$ & P-Value \\
\hline Constante & -2.06599 & 23.14689 & -0.089256 & 0.9306 \\
\hline$y_{t}$ & 0.462354 & 5.85503 & 0.07896 & 0.9386 \\
\hline$y_{t}^{2}$ & -0.025565 & 0.37057 & -0.06898 & 0.9464 \\
\hline$H_{t}$ & 0.117940 & 0.13633 & 0.86504 & 0.4073 \\
\hline$R g_{t}$ & -0.010589 & 0.02336 & -0.45323 & 0.6601 \\
\hline$\pi_{t}$ & 0.215873 & 0.07820 & 2.76041 & 0.0201 \\
\hline$I_{t}$ & 0.001224 & 0.01558 & 0.07856 & 0.9389 \\
\hline$P_{t-1}$ & 0.756349 & 0.10136 & 7.46131 & 0 \\
\hline \multicolumn{5}{|r|}{} \\
\hline
\end{tabular}

\begin{tabular}{|c|c|c|c|c|}
\hline \multicolumn{5}{|c|}{$R_{t}=\beta_{0}+\beta_{1} y_{t}+\beta_{2} y_{t}^{2}+\beta_{3} H_{t}+\beta_{4} R g_{t}+\beta_{5} \pi_{t}+\beta_{6} I_{t}+\beta_{7} R_{t-1}+u_{t}$} \\
\hline Variable & Coeficiente & Error Estándar & Estadístico $t$ & P-Value \\
\hline Constante & -95.43629 & 67.74224 & -1.408815 & 0.1892 \\
\hline$y_{t}$ & 24.10245 & 17.10134 & 1.409389 & 0.1891 \\
\hline$y_{t}^{2}$ & -1.514335 & 1.079406 & -1.402934 & 0.1909 \\
\hline$H_{t}$ & -0.769233 & 0.465163 & -1.653686 & 0.1292 \\
\hline$R g_{t}$ & -0.066996 & 0.095051 & -0.704845 & 0.4970 \\
\hline$\pi_{t}$ & -0.315404 & 0.298458 & -1.056777 & 0.3155 \\
\hline$I_{t}$ & 0.021246 & 0.060174 & 0.353081 & 0.7314 \\
\hline$R_{t-1}$ & -0.037073 & 0.311405 & -0.119049 & 0.9076 \\
\hline \multicolumn{5}{|c|}{$R^{2}=0.78502$} \\
\hline
\end{tabular}

Notas: Muestra (ajustada) del primer trimestre de 1998 al segundo trimestre del 2002, el número de observaciones es de 18 y las desviaciones estándar se calcularon usando la matriz de varianzas y covarianzas Newey-West HAC.

\footnotetext{
1 Las relaciones son muy débiles cuando se usa la medida de riesgo $R$.
} 


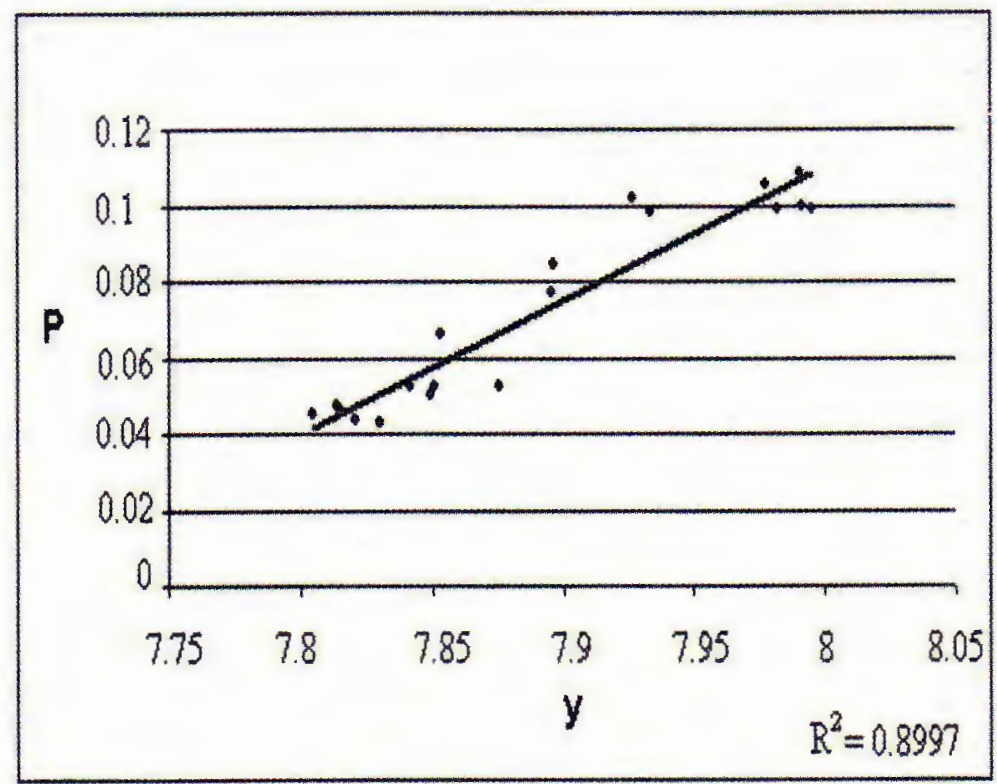

Gráfico 5. Cártera vencida vs. Tamaño.

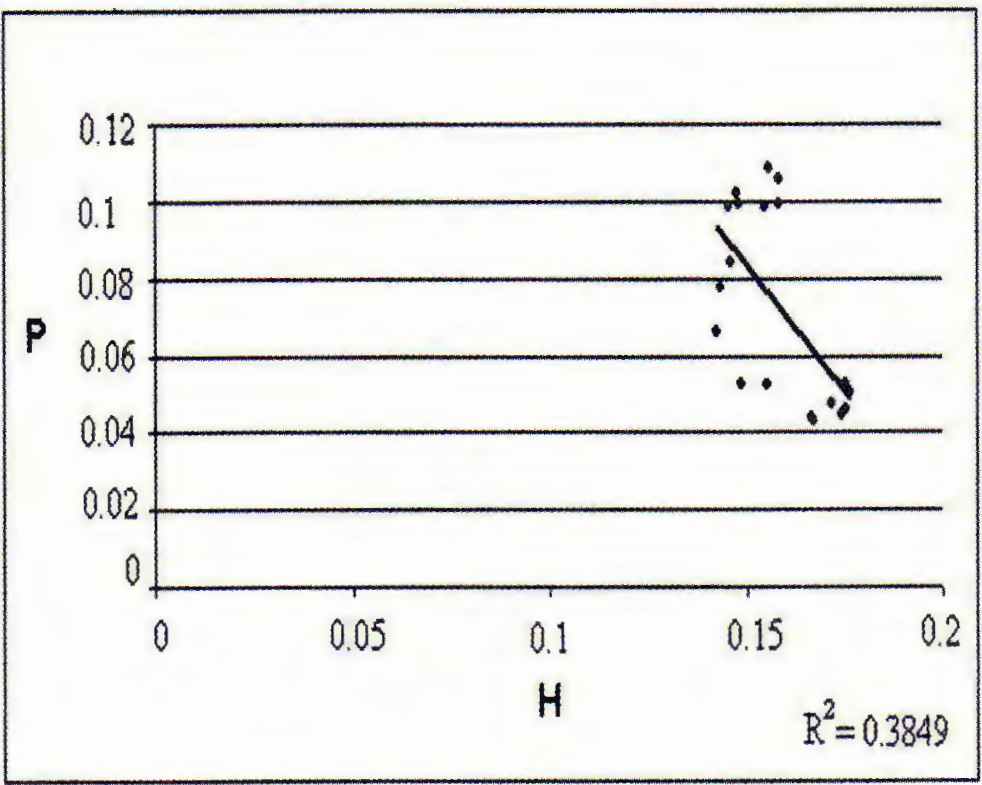

Gráfico 6. Cártera vencida vs. Concentración. 


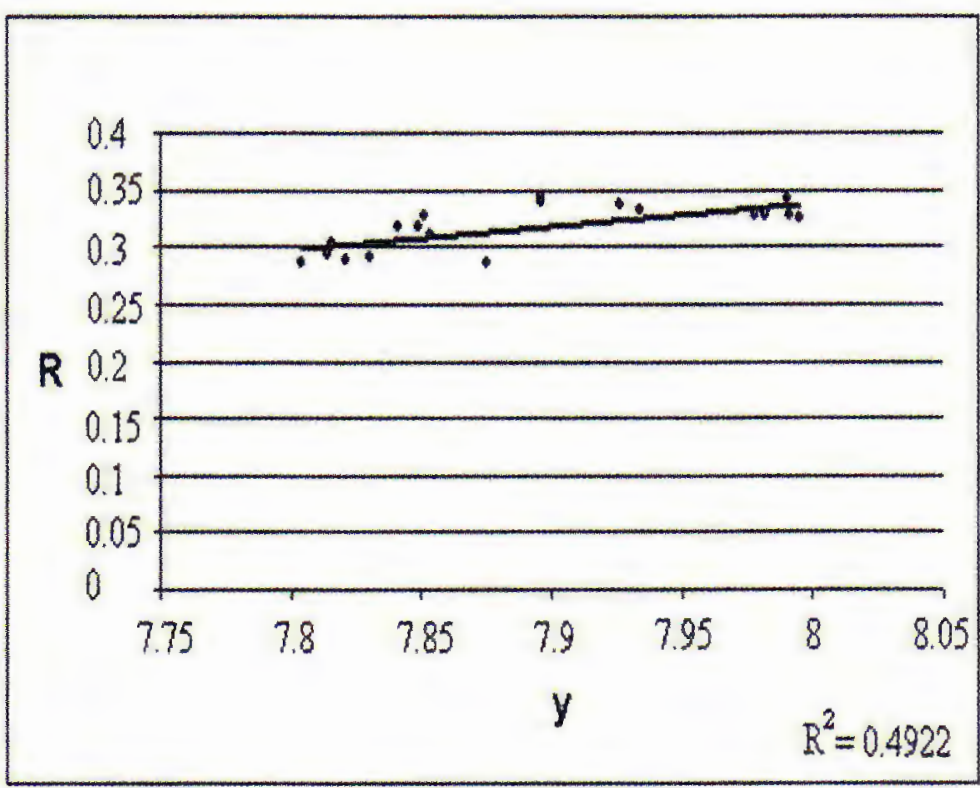

Gráfico 7. Índice de calificación vs. Tamaño.

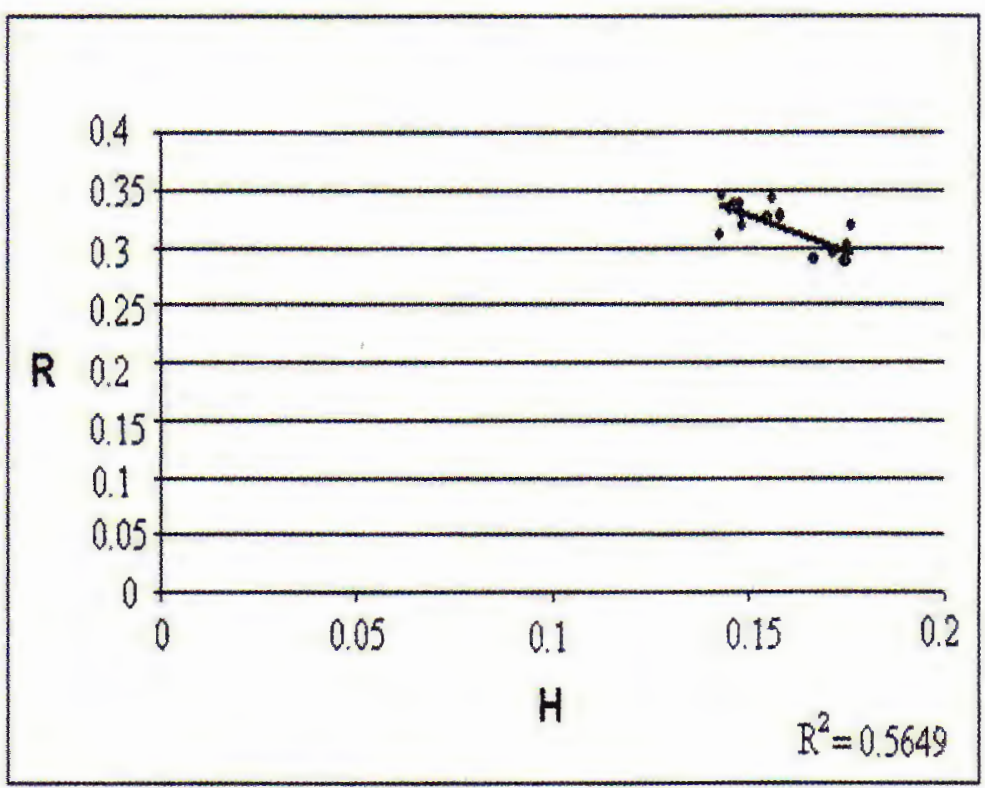

Gráfico 8. Índice de calificación vs. Concentración. 


\subsubsection{Datos Panel}

En esta parte se estiman ecuaciones similares a las presentadas en la sección anterior. Aquí, tampoco las variables resultaron significativas. No existe una relación clara de dependencia entre las variables de riesgo, tamaño y nivel de concentración. En las gráficas 9-12 no se observa patrón de correlación.

\begin{tabular}{|c|c|c|c|c|}
\hline \multicolumn{5}{|c|}{$\begin{aligned} P_{i, t}= & \beta_{0}+\beta_{1} y_{i, t}+\beta_{2} y_{i, t}^{2}+\beta_{3} S_{i, t}+\beta_{4} H_{i, t}+\beta_{5} R g_{i, t}+\beta_{6} \pi_{i, t}+\beta_{7} I_{i, t} \\
& +\beta_{8} P_{i, t-1}+u_{i, t}\end{aligned}$} \\
\hline Variable & Coeficiente & Error Estándar & Estadístico $t$ & P-Value \\
\hline Constante & -0.037185 & 0.026471 & -1.404709 & 0.1607 \\
\hline$y_{t}$ & 0.008460 & 0.007343 & 1.152118 & 0.2498 \\
\hline$y_{t}^{2}$ & -0.002748 & 0.001816 & -1.513129 & 0.1308 \\
\hline$S_{t}$ & 0.257172 & 0.173309 & 1.483889 & 0.1384 \\
\hline$H_{t}$ & 0.246862 & 0.157255 & 1.569821 & 0.1170 \\
\hline$R g_{t}$ & 0.035817 & $0.054 ? 81$ & 0.658628 & 0.5104 \\
\hline$\pi_{t}$ & 0.086698 & 0.080272 & 1.080049 & 0.2806 \\
\hline$I_{t}$ & 0.002803 & 0.019145 & 0.146413 & 0.8836 \\
\hline$P_{t-1}$ & 0.924289 & 0.127681 & 7.239075 & 0.0000 \\
\hline \multicolumn{4}{|c|}{$R^{\frac{1}{2}}=0.694538$} & $D W=1.92969$ \\
\hline
\end{tabular}

\begin{tabular}{|c|c|c|c|c|}
\hline \multicolumn{5}{|c|}{$\begin{aligned} R_{i, t}= & \beta_{0}+\beta_{1} y_{i, t}+\beta_{2} y_{i, t}^{2}+\beta_{3} S_{i, t}+\beta_{4} H_{i, t}+\beta_{5} R g_{i, t}+\beta_{6} \pi_{i, t}+\beta_{7} I_{i, t} \\
& +\beta_{8} R_{i, t-1}+u_{i, t}\end{aligned}$} \\
\hline Variable & Coeficiente & Error Estándar & Estadístico $t$ & P-Value \\
\hline Constante & 0.052744 & 0.046485 & 1.134644 & 0.2570 \\
\hline$y_{t}$ & -0.000173 & 0.003854 & -0.044933 & 0.9642 \\
\hline$y_{t}^{2}$ & 0.000374 & 0.000873 & 0.428467 & 0.6685 \\
\hline$S_{t}$ & -0.045792 & 0.084773 & -0.540173 & 0.5893 \\
\hline$H_{t}$ & -0.100879 & 0.190308 & -0.530084 & 0.5963 \\
\hline$R g_{t}$ & -0.053088 & 0.054614 & -0.972063 & 0.3314 \\
\hline$\pi_{t}$ & -0.139318 & 0.208974 & -0.666677 & 0.5053 \\
\hline$I_{t}$ & 0.052558 & 0.034087 & 1.541890 & 0.1237 \\
\hline$R_{t-1}$ & 0.848643 & 0.084792 & 10.00848 & 0.0000 \\
\hline & & & 0.726743 & $=1.924274$ \\
\hline
\end{tabular}

Notas: Muestra (ajustada) del primer trimestre de 1998 al segundo trimestre del 2002, el número de secciones cruzadas utilizadas es de 34 , el número de observaciones totales en el panel es de 562 y las desviaciones estándar se calcularon usando la matriz de varianzas y covarianzas de White. 


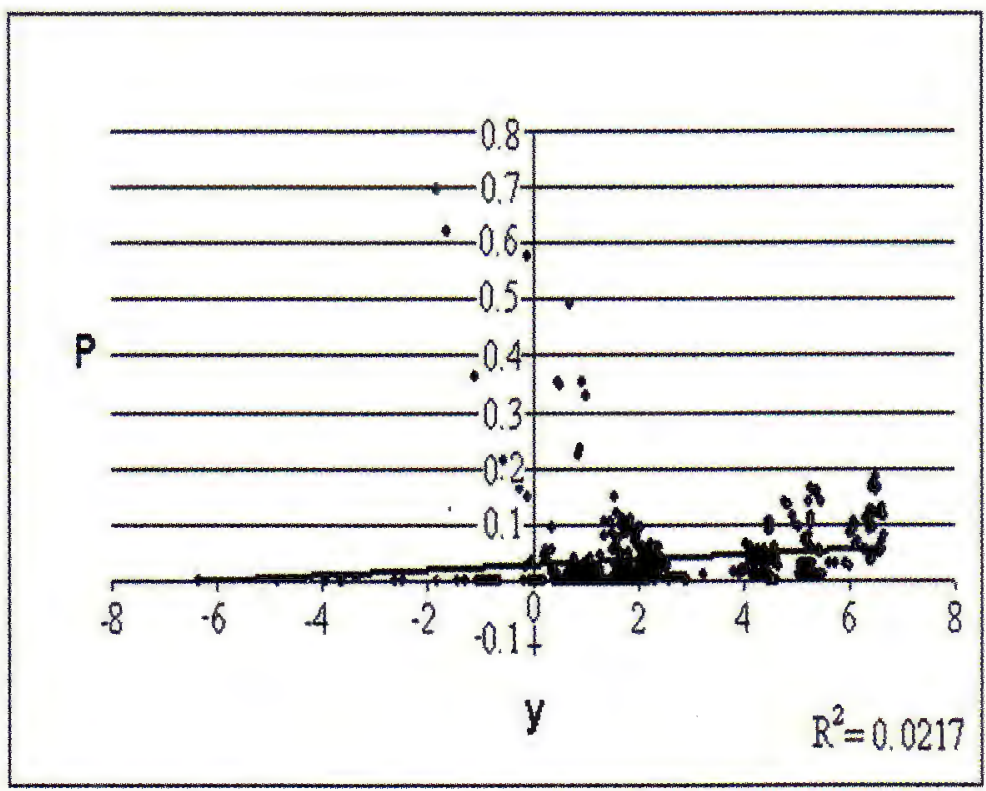

Gráfico 9. Cártera vencida vs. Tamaño.

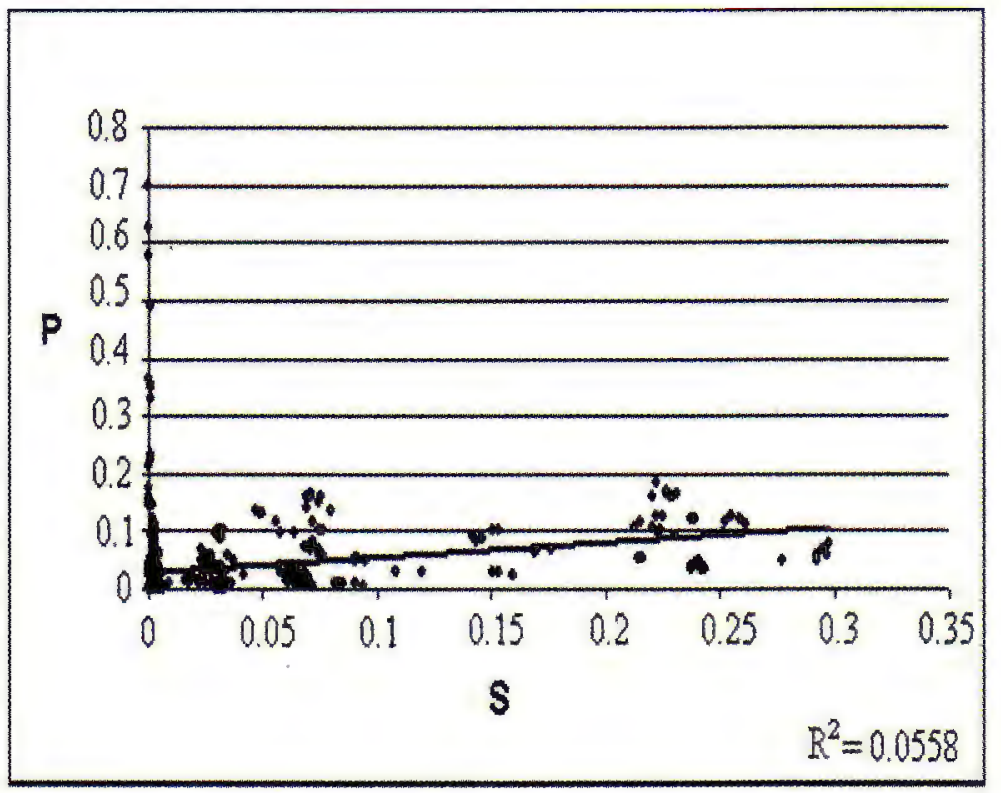

Gráfico 10. Cártera vencida vs. Participación. 


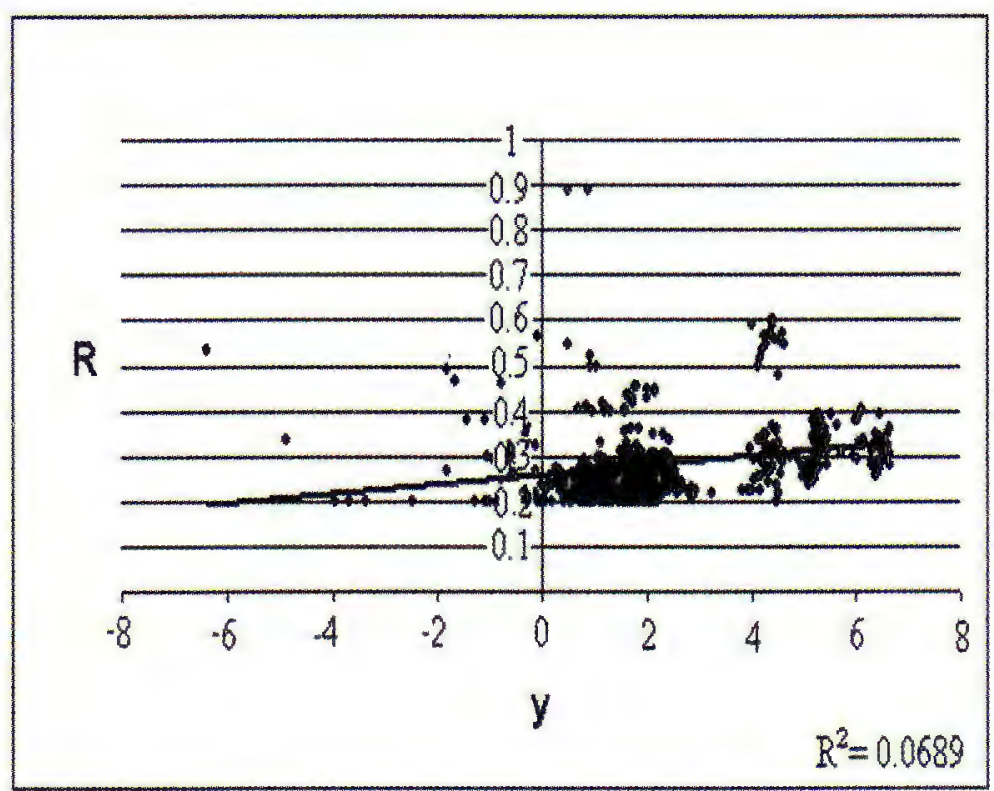

Gráfico 11. Índice de calificación vs. Tamaño.

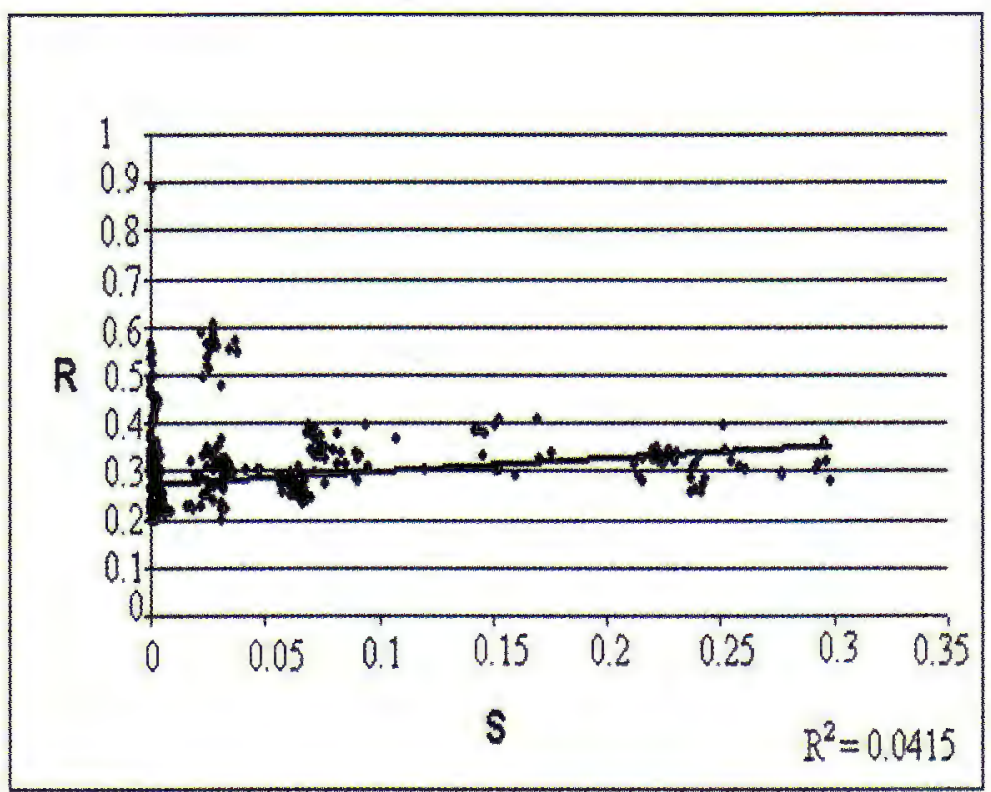

Gráfico 12. Índice de calificación vs. Participación. 


\section{Conclusiones}

En este documento se ha probado empíricamente la supuesta relación entre el tamaño de los bancos, la concentración y el riesgo. Esta relación no es trivial, ya que puede existir una correlación positiva o negativa entre el tamaño y riesgo dependiendo del modelo que se use. En este trabajo se utilizaron datos del sistema bancario mexicano entre el año de 1997 al 2002 para verificar que relación se cumple para México. De acuerdo con nuestro análisis no es posible establecer claramente una dependencia positiva y/o negativa entre las variables consideradas.

\section{Bibliografía}

Bhattacharya, S., A. Boot, and A. Thakor (1998). The Economics of Bank Regulation. Journal of Money, Credit, and Banking, (30)4, pp. 745-770.

Boyd, J. and M. Gertler (1994). The Role of Large Banks in the Recent U. S. Banking Crisis. Federal Reserve of Minneapolis Quarterly Review, (18)1.

Boyd, J. and S. Graham (1996). Consolidation in U. S. Banking: Implications for Efficiency and Risk. Working Paper. Federal Reserve Bank of Minneapolis.

Boyd, J. and D. Runkle (1993). Size and Performance of Banking Firms. Journal of Monetary Economics, Vol. 31, pp. 47-67.

Chumacera, R. y P. Langoni (2001). Riesgo, Tamaño y Concentración en el Sistema Bancario Chileno, (4)1, pp. 25-33.

Demsetz, R. and P. Strahan (1995). Historical Patterns and Recent Changes in the Relationship between Bank Holding Company Size and Risk. Economic Policy Review, July, pp. $13-26$.

Levine, R. (2000). Bank Concentration: Chile and International Comparisons. Documentos de Trabajo. Banco Central de Chile.

Strahan, P. (1999). Borrower Risk and the Price Nonprice Terms of Bank Loans. Working Paper. Federal Reserve Bank of New York. 